\title{
Incidence of Periodontal Disease among Adolescents
}

\author{
Delia-Roxana Dicu¹, Ana Petra Lazăr², Luminița Lazăr³ \\ 1 Faculty of Dental Medicine, "George Emil Palade" University of Medicine, Pharmacy, Science and Technology, Târgu Mureș, Romania \\ 2 Department of Oral Rehabilitation and Occlusology, Faculty of Dental Medicine, "George Emil Palade" University of Medicine, \\ Pharmacy, Science and Technology, Târgu Mureș, Romania \\ 3 Department of Periodontology and Oral Diagnosis, Faculty of Dental Medicine, "George Emil Palade" University of Medicine, \\ Pharmacy, Science and Technology, Târgu Mureș, Romania
}

\section{CORRESPONDENCE}

Ana Petra Lazăr

Str. Gheorghe Marinescu nr. 38

540139 Târgu Mureș, Romania

Tel: +40 265215551

E-mail: analazar_mvfa@yahoo.com

\section{ARTICLE HISTORY}

Received: May 28, 2020

Accepted: June 15, 2020
Delia-Roxana Dicu • Str. Gheorghe Marinescu nr. 38, 540139 Târgu Mureș, Romania. Tel: +40 748864 900, E-mail: dicu.delia@yahoo.com

Luminita Lazăr • Str. Gheorghe Marinescu nr. 38 , 540139 Târgu Mureș, Romania. Tel: +40 265215 551, E-mail: luminita.lazar15@gmail.com

\section{ABSTRACT}

Background: Despite the scarcity of studies regarding periodontal disease in young patients (teenagers and young adults), it seems that this disorder is also affecting the young population. Risk factors for periodontal disease include older age, chronic tobacco use, male gender, habits regarding oral hygiene, educational status, ethnicity, and financial status. Aim: This study aimed to evaluate the periodontal health among adolescents in two high schools in Târgu Mureș that had dental practices. Material and Methods: An online questionnaire consisting of 16 questions was distributed among high schoolers of Târgu Mureș. Data about personal characteristics, oral hygiene habits, family history of periodontal disease, risk factors for periodontal disease, and symptoms of periodontal disease observed by the respondents were collected and analyzed. Results: Out of the 501 teenagers who responded to the online questionnaire, $114(22.8 \%)$ were 18 years old and were mostly females (88.2\%). Regarding oral hygiene habits, 75.8\% prefer a manual toothbrush over an electric toothbrush, $66.7 \%$ brush their teeth twice a day, and $54.1 \%$ practice a horizontal method of toothbrushing. Mouthwash was the most used oral hygiene aid (58.3\%). Family history of periodontal disease was observed in $21.9 \%$ of respondents. As favoring factors, nicotine addiction (23.8\%), bruxism (24.4\%), interposition of various objects between teeth (48.3\%), past or present orthodontic treatments (38.7\%) were recorded. Symptoms of gingivitis and periodontitis, such as gingival bleeding (81.4\%), redness of gingiva (39.3\%), increased gingival volume (44.5\%), gingival retraction (22.8\%), and halitosis (81\%), were present in the responding teenagers. Conclusions: In this study, periodontal disease was affecting mostly adolescent females who are practicing inappropriate methods of toothbrushing with inadequate frequency.

Keywords: periodontal health, adolescents, gingivitis, periodontitis 


\section{INTRODUCTION}

Nowadays, the major focus of dental medicine is maintaining a lifelong healthy state of the hard and soft tissues. In order to achieve this goal, periodontal diseases should be diagnosed early in life, starting from childhood and adolescence, as they affect more than half of the teenage population, which are mainly asymptomatic. Bimstein (1991) stressed the importance of prevention, early diagnosis, and early treatment of periodontal diseases in children and adolescents, because the prevalence and severity of periodontal diseases is high. Also, the incipient periodontal diseases found in childhood may develop into advanced periodontal diseases during adulthood, and there is a direct association between periodontal disease and systemic conditions. Patients, families, or populations at risk could be identified and included in special prevention or treatment programs, as prevention and treatment of most periodontal diseases is relatively simple and very effective, providing lifetime benefits. ${ }^{1,2}$

This study aimed to evaluate periodontal health among adolescents in two high schools in Târgu Mureș that had a dental practice.

\section{MATERIAL AND METHODS}

The study was initially created as a clinical trial involving the clinical examination of teenagers from "Alexandru Papiu Ilarian" National College and "Traian Săvulescu” Agricultural College that had a dental practice. We determined that 10th and 11th graders were appropriate to be examined. The first step included a presentation concerning periodontal health and a questionnaire about personal characteristics, oral hygiene habits, family history of periodontal disease, favoring factors for periodontal disease, and symptoms of periodontal disease. Then, the next stage included an objective clinical examination in school dental practices using dental examination instruments (probe, mirror, tweezers) and a periodontal probe. We examined plaque index, tartar index, gingival index, clinical attachment loss, papillary bleeding index, mobility, furcation involvement, and periodontal probing depth on Ramfjord teeth. The study was approved by the Ethics Committee of "George Emil Palade" University of Medicine and Pharmacy, Science and Technology of Târgu Mureș was obtained. All participants gave informed consent for participation in this study and all the study procedures were conducted in accordance with the principles stated in the Declaration of Helsinki.

Due to the worldwide epidemiologic context of the time when we were about to start the study (March 2020), a safe clinical examination was not possible; therefore, we adjusted accordingly and narrowed the study down to an online questionnaire. Thus, the present study was based on a questionnaire consisting of 16 questions that was distributed among the high schoolers of Târgu Mureș. When formulating the questions, we used colloquial language in order to have a greater addressability. Data about personal characteristics, oral hygiene habits, family history of periodontal disease, risk factors for periodontal disease, and symptoms of periodontal disease observed by the respondents were collected and analyzed.

The resulting data were collected and interpreted using Google Forms and Microsoft Excel.

\section{RESULTS}

Data from 501 responding adolescents were collected and analyzed. The respondents were aged between 14 and 19 years, most of them being 18 years old. The majority of the respondents (88.2\%) were female. Regarding oral hygiene habits, $75.8 \%$ stated that they prefer a manual toothbrush over an electric toothbrush, $66.7 \%$ brush their teeth twice a day, and $54.1 \%$ practice a horizontal method of toothbrushing. Mouthwash was the most used oral hygiene aid (58.3\%). Data about oral hygiene habits is presented in Table 1.

Family history of periodontal disease was observed in $21.9 \%$ of the study population.

Favoring factors that play an important role in the development of periodontal diseases were also addressed in the questionnaire. The answers revealed that $23.8 \%$ of the participants were smokers, $24.4 \%$ practiced the habit of bruxism, $48.3 \%$ had viciously interposing objects between their teeth, and $38.7 \%$ were undergoing an orthodontic treatment.

Given the lack of an objective clinical examination, we focused some of the questions on the symptoms of gingivitis and periodontitis in order to have a general view regarding these pathologies. When asked about gingival bleeding, $81.4 \%$ of the respondents answered positively. A similar percentage of participants $(81 \%)$ admitted to have halitosis, and $39.3 \%$ have observed redness of the gingiva. Increased gingival volume and gingival retractions were observed by $44.5 \%$ and $22.8 \%$ of respondents, respectively.

\section{DISCUSSIONS}

Despite the scarcity of studies regarding periodontal disease in young patients (teenagers and young adults), it seems that this disorder is also affecting the young popula- 
TABLE 1. Distribution of oral hygiene habits among the responding adolescents

\begin{tabular}{llc}
\hline Question & Possible answers & Answered yes (\%) \\
\hline Type of toothbrush & Manual & 75.8 \\
\multirow{2}{*}{ Daily toothbrushing frequency } & Electric & 24.2 \\
& Does not brush on a daily basis & 0 \\
& Once a day & 33.1 \\
Toothbrushing method & Twice a day & 66.7 \\
\multirow{2}{*}{ Auxiliary method of removing } & Horizontal & 54.1 \\
dental plaque & Vertical & 45.9 \\
& Mouthwash & 58.3 \\
& Interdental floss & 34.1 \\
& Wooden toothpick & 29.1 \\
& Oral irrigator & 13 \\
& Interdental toothbrush & 12 \\
\hline
\end{tabular}

tion. Risk factors for periodontal disease include older age, chronic tobacco use, male gender, habits regarding oral hygiene, educational status, ethnicity, and financial status. ${ }^{3,4}$ In the present study, we aimed to assess the prevalence of periodontal disease in the high school population of a medium-sized city in Central Romania. Although initially designed as a cross-sectional clinical study, the global COVID-19 pandemic situation has led to a drastic change in the research methodology and study design. Therefore, we conducted a questionnaire-based study on the clinical signs and symptoms of periodontal disease, and also its associated risk factors, on a population of young students aged between 14 and 19 years, from two high schools in Târgu Mureș.

Given the lack of an objective clinical examination, we considered the triad of gingival bleeding - increased gingival volume - redness of gingiva as an indicator of gingivitis, regardless of the causal factor (dental plaque, hormones, manifestation of general disease etc.). Gingivitis was present in a relatively large proportion of respondents (26.5\%). Moreover, we considered the simultaneous presence of halitosis and gingival retraction as indicator of periodontitis, which was present in a lower rate (19.3\%). The gender distribution of subjects with periodontitis and gingivitis in our study population was skewed towards female respondents, which was similar to a study conducted in Bucharest. On the other hand, two international studies found that males presented higher odds for periodontal disease compared to females. 5,6

Regarding the age of the participants, there was a tendency for the prevalence of gingivitis and periodontitis to increase with age. The increasing trend of gingivitis and periodontitis during puberty and adolescence could be related to hormonal influences over dental plaque and gingi- val tissue. ${ }^{7,8}$ Daily toothbrushing frequency was similar for both individuals who use a manual toothbrush or an electric toothbrush. However, regardless of the type of toothbrush used, the percentage of teenagers who brush their teeth twice a day was almost double compared to those who brush their teeth once a day. The efficiency of toothbrushing was also assessed. Gingivitis was more frequent (36.1\%) among respondents who brush their teeth once a day and among those who use an electric toothbrush (28.9\%). The latter result is inconsistent with other studies that report an increased dental plaque removal using an electric toothbrush by $4 \%$ to $29.6 \% .^{9-11}$

By adolescence, people should grasp the correct technique of toothbrushing, which is the vertical one. Most of the respondents were using a horizontal technique, which demonstrates the lack of oral hygiene education. The main consequence of horizontal toothbrushing is gingival retraction, which is also directly related to bristle hardness, the duration of each toothbrushing session, as well as the frequency of changing a used toothbrush. ${ }^{12,13}$ Adolescents seem to show a great interest in auxiliary methods of removing dental plaque, using mostly mouthwash, interdental floss, and wooden toothpicks, although they are not proven to decrease dental plaque; only interdental brushes showed a decrease in dental plaque (32\%) and gingivitis $(34 \%) .{ }^{14-16}$

Gingivitis was predominant among respondents who reported a family history of periodontal disease, although other studies did not confirm whether the disease has a genetic transmission or it is a result of environmental factors. ${ }^{17,18}$

According to the World Health Organization, nicotine addiction exerts a great influence on oral health, being frequently associated with a poor oral hygiene and the pres- 
ence of red complex periodontal pathogens. ${ }^{19,20}$ Although most of the international studies agree that most smokers are males, our study shows an increased percentage of female smokers ( $25.4 \%$ vs. $23.5 \%)$ and consequently, an increased percentage of gingivitis and halitosis. Other major contributing factors to gingivitis and gingival retraction are bruxism and the vicious habit of interposing various objects between teeth, which were relatively frequent in the questioned population. It is proven that nocturnal bruxism is present in children and adolescents more often than in adults, but it does not cause gingivitis or gingival retraction without the presence of dental plaque..$^{2,21-24}$

Orthodontic materials increase the retentivity of the dental arch and, consequently, dental plaque build-up and the development of periodontal disease. Gingivitis was more frequent in teenagers with orthodontic treatment (34.5\%) in our study. This result is supported by other studies which state that dental plaque build-up and hyperplastic gingivitis occur in the first two months after starting the orthodontic treatment. ${ }^{25,26}$ Patients treated with removable materials showed a decreased plaque index and gingivitis due to superior cleaning possibilities compared to fixed appliances. ${ }^{27}$ Gingival retractions are also increased in teenagers with orthodontic treatment (24.2\%), even though they are not specific to this age group..$^{28}$

\section{Study limitations}

The major limitation of this study was the difficulty to conduct a more objective clinical examination, in the absence of which the results have only an indicative value. Other sources of error could be misinterpretation of symptoms by the respondents or truncated information, and misinterpretation of results. The study supports further additions and improvements by adding a clinical examination as it was initially intended.

Such studies are of great value in the current clinical activity because they evaluate the state of the general population, and through these studies, health education programs focused on oral health can be implemented. Furthermore, epidemiologic studies justify and support therapeutic protocols by highlighting the risk factors for the evaluated disorder.

\section{CONCLUSIONS}

In this study, periodontal disease was affecting mostly adolescent females who are practicing inappropriate methods of toothbrushing with inappropriate frequency. Although the respondents were keen on using auxiliary methods of removing dental plaque, their effectiveness has not been demonstrated. Bruxism is a major favoring factor for periodontal disease due to an increased prevalence of gingivitis and gingival retraction among adolescents that practice this habit. Another favoring factor, nicotine addiction, can cause gingivitis and halitosis among female adolescents. Orthodontic appliances cause increased dental plaque retention leading to gingivitis and gingival retraction.

\section{CONFLICT OF INTEREST}

Nothing to declare.

\section{REFERENCES}

1. Oh TJ, Eber R, Wang HL. Periodontal diseases in the child and adolescent. J Clin Periodontol. 2002;29:400-410.

2. Dumitriu HT, Dumitriu S, Dumitriu AS. Tratat de parodontologie. Bucharest Ed. Viața Medicală Românească, 2015; p. 112-148.

3. Peeran SW, Singh AJ, Alagamuthu G, Naveen Kumar PG. Periodonta status and its risk factors among young adults of the Sebha city (Libya). Dent Res J (Isfahan). 2013;10:533-538.

4. Periodontal Disease in Adults (Age 20 to 64). Available at: https://www nidcr.nih.gov/research/data-statistics/periodontal-disease/adults

5. Eke PI, Dye BA, Wei L, Thornton-Evans GO, Genco RJ. CDC Periodontal Disease Surveillance workgroup: James Beck (University of North Carolina, Chapel Hill, USA), Gordon Douglass (Past President, American Academy of Periodontology), Roy Page (University of Washin. Prevalence of periodontitis in adults in the United States: 2009 and 2010. J Dent Res. 2012;91:914-920.

6. Grossi SG, Genco RJ, Machtei EE, et al. Assessment of risk for periodontal disease. II. Risk indicators for alveolar bone loss. J Periodontol. 1995;66:23-29.

7. Dietrich T, Kaye EK, Nunn ME, Van Dyke T, Garcia RI. Gingivitis susceptibility and its relation to periodontitis in men. J Dent Res. 2006;85:1134-1137.

8. Bimstein E, Matsson L. Growth and development considerations in the diagnosis of gingivitis and periodontitis in children. Pediatr Dent 1999;21:186-191.

9. Elkerbout TA, Slot DE, Rosema NAM, Van der Weijden GA. How effective is a powered toothbrush as compared to a manual toothbrush? A systematic review and meta-analysis of single brushing exercises. Int J Dent Hyg. 2020;18:17-26.

10. Sicilia A, Arregui I, Gallego M, Cabezas B, Cuesta S. A systematic review of powered vs manual toothbrushes in periodontal cause-related therapy. J Clin Periodontol. 2002;29 Suppl3:39-91.

11. Bartizek RD, Biesbrock AR. Dental plaque removal efficacy of a batterypowered toothbrush vs. a control Japanese manual toothbrush. Am J Dent. 2002;15:33A-36A.

12. Heasman PA, Holliday R, Bryant A, Preshaw PM. Evidence for the occurrence of gingival recession and non-carious cervical lesions as a consequence of traumatic toothbrushing. J Clin Periodontol. 2015;42Suppl16:S237-S255.

13. Bergström J, Lavstedt S. An epidemiologic approach to toothbrushing and dental abrasion. Community Dent Oral Epidemiol. 1979;7:57-64.

14. Worthington HV, MacDonald L, Poklepovic Pericic T, et al. Home use of interdental cleaning devices, in addition to toothbrushing, for preventing and controlling periodontal diseases and dental caries. Cochrane Database Syst Rev. 2019;4:CD012018.

15. Sälzer S, Slot DE, Van der Weijden FA, Dörfer CE. Efficacy of inter-denta mechanical plaque control in managing gingivitis - a meta-review. J Clin Periodontol. 2015;42Suppl 16:S92-S105.

16. Richards D. The effectiveness of interproximal oral hygiene aids. Evid Based Dent. 2018;19:107-108.

17. Tatakis DN, Trombelli L. Modulation of clinical expression of plaqueinduced gingivitis. I. Background review and rationale. J Clin Periodontol. 2004;31:229-238 
18. Michalowicz BS, Diehl SR, Gunsolley JC, et al. Evidence of a substantial genetic basis for risk of adult periodontitis. J Periodontol. 2000;71:16991707.

19. Genco RJ, Borgnakke WS. Risk factors for periodontal disease. Periodontol 2000. 2013:62:59-94

20. Honkala S, Honkala E, Newton T, Rimpelä A. Toothbrushing and smoking among adolescents - aggregation of health damaging behaviours. J Clin Periodontol. 2011;38:442-448.

21. Melo G, Duarte J, Pauletto P, et al. Bruxism: An umbrella review of systematic reviews. J Oral Rehabil. 2019;46:666-690.

22. Bosnjak A, Vućićević-Boras V, Miletić I, Bozić D, Vukelja M. Incidence of oral habits in children with mixed dentition. J Oral Rehabil. 2002;29:902-905.

23. Restrepo CC, Tirado M, Jimenez KJ. Association of sleep bruxism and dental plaque factors on signs of periodontal disease in children in the mixed dentition. Int J Paediatr Dent. 2016;26:477-485
24. Manfredini D, Ahlberg J, Mura R, Lobbezoo F. Bruxism is unlikely to cause damage to the periodontium: findings from a systematic literature assessment. J Periodontol. 2015;86:546-555.

25. Cardoso-Silva C, Barbería E, Ramos Atance JA, Maroto M, Hernández A, García-Godoy F. Microbiological analysis of gingivitis in pediatric patients under orthodontic treatment. Eur J Paediatr Dent. 2011;12:210-214.

26. Alhaija ESA, Al-Saif EM, Taani DQ. Periodontal health knowledge and awareness among subjects with fixed orthodontic appliance. Dental Press J Orthod. 2018;23:40.e1-40.e9.

27. Abbate GM, Caria MP, Montanari P, et al. Periodontal health in teenagers treated with removable aligners and fixed orthodontic appliances. $J$ Orofac Orthop. 2015;76:240-250.

28. Alhaija ESA, Al-Saif EM, Taani DQ. Periodontal health knowledge and awareness among subjects with fixed orthodontic appliance. Dental Press J Orthod. 2018;23:40.e1-40.e9. 